PROCEEDINGS OF THE

AMERICAN MATHEMATICAL SOCIETY

Volume 140, Number 6, June 2012, Pages 1997-2001

S 0002-9939(2011)11247-4

Article electronically published on September 30, 2011

\title{
SYNDETIC SETS AND AMENABILITY
}

\author{
VERN I. PAULSEN
}

(Communicated by Thomas Schlumprecht)

\begin{abstract}
We prove that if an infinite, discrete semigroup has the property that every right syndetic set is left syndetic, then the semigroup has a left invariant mean. We prove that the weak*-closed convex hull of the two-sided translates of every bounded function on an infinite discrete semigroup contains a constant function. Our proofs use the algebraic properties of the Stone-Cech compactification.
\end{abstract}

\section{THE MAIN THEOREMS}

Let $S$ be an infinite discrete semigroup. Given $f \in \ell^{\infty}(S)$ and $t \in S$ set $(t \cdot f)(s)=$ $f^{t}(s)=f(s t)$ and $r_{t}(f)=f^{t}$, which is called the right translate of $f$ by $t$. Similarly, we set $(f \cdot t)(s)=f_{t}(s)=f(t s)$ and $l_{t}(f)=f_{t}$, which is called the left translate of $f$ by $t$. Moreover, $t \rightarrow t \cdot f$ and $t \rightarrow f \cdot t$ define associative, left and right actions of $S$ on $\ell^{\infty}(S)$.

A classic result of Mitchell 5 says that the weak*-closed convex hull of the set of right(respectively, left) translates of $f$ contains a constant function for every $f \in \ell^{\infty}(S)$ if and only if $\ell^{\infty}(S)$ has a left (respectively, right) invariant mean. A semigroup is called left (respectively, right) amenable if $\ell^{\infty}(S)$ has a left (respectively, right) invariant mean. For a simpler proof of Mitchell's result, along with an extension to the topological case, see the paper of Granirer and Lau [3].

M. M. Day proved 2 that if a semigroup has a left invariant mean and a right invariant mean, then it has a mean that is simultaneously left and right invariant, called an invariant mean. A semigroup with an invariant mean is called amenable. By another result of Day [2, any group that has a left or right invariant mean has an invariant mean. However, Day also gave examples of semigroups that had left (respectively, right) invariant means but no right (respectively, left) invariant means.

In this paper we prove the following theorems.

Theorem 1. Let $S$ be an infinite discrete semigroup, let $f \in \ell^{\infty}(S)$, and let $\mathcal{C}(f)$ denote the weak ${ }^{*}$-closed, convex hull of the set of functions obtained by taking right and left translates of $f$. Then $\mathcal{C}(f)$ contains a constant function.

While there might be many ways to see this result, the proof we will present uses syndetic sets and the algebra in the Stone-Cech compactification. This proof also leads to a new condition for amenability involving syndetic sets.

Received by the editors February 2, 2011.

2010 Mathematics Subject Classification. Primary 43A07; Secondary 22A15.

This research was supported in part by NSF grant DMS-0600191.

(C)2011 American Mathematical Society 1997

Reverts to public domain 28 years from publication 
Recall that a subset $B$ of a semigroup $S$ is called right syndetic [4] if there is a finite set $\left\{t_{1}, \ldots, t_{k}\right\}$ of $S$ such that $S=t_{1}^{-1} B \cup \cdots \cup t_{k}^{-1} B$, where $t^{-1} B=\{s \in$ $S: t s \in B\}$. A set $B$ is called left syndetic [4 if there is a finite set $\left\{t_{1}, \ldots, t_{k}\right\}$ in $S$ such that $S=B t_{1}^{-1} \cup \cdots \cup B t_{k}^{-1}$, where $B t^{-1}=\{s \in S: s t \in B\}$. When authors speak of syndetic sets with no reference to right or left, they generally mean right syndetic.

Theorem 2. Let $S$ be an infinite, discrete semigroup. If every right (respectively, left) syndetic subset of $S$ is also left (respectively, right) syndetic and $f \in \ell^{\infty}(G)$, then the weak*-closed convex hull of the set of right (respectively, left) translates of $f$ contains a constant function.

Combining this theorem with Mitchell's result, we have:

Corollary 3. Let $S$ be an infinite discrete semigroup. If every right (respectively, left) syndetic set in $S$ is left (respectively, right) syndetic, then there is a left (respectively, right) invariant mean on $\ell^{\infty}(S)$. If every right syndetic set is left syndetic and every left syndetic set is right syndetic, then $S$ is amenable.

Corollary 4. Let $G$ be an infinite discrete group. If $G$ is not amenable, then $G$ contains a set that is right syndetic but not left syndetic.

Remark 5. In the case of a group, it is easily seen that a set $B$ is right (respectively, left) syndetic if and only if $B^{-1}$ is left (respectively, right) syndetic. Thus, every right syndetic set is left syndetic if and only if $B^{-1}$ is right syndetic for every right syndetic set $B$. It also follows for groups that every right syndetic set is left syndetic if and only if every left syndetic set is right syndetic.

Remark 6. Although the property that every right syndetic set is left syndetic implies amenability for groups, this property does not characterize amenability. The free product of the group of order two with itself, $\mathbb{Z}_{2} * \mathbb{Z}_{2}$, is amenable but contains a right syndetic subset that is not left syndetic. Indeed, if we let the two generators of this free product be denoted $a, b$, and let $B$ be the subset of all words beginning with $b$, then $\mathbb{Z}_{2} * \mathbb{Z}_{2}=B \cup a B$, but $B$ is not left syndetic. To see that $\mathbb{Z}_{2} * \mathbb{Z}_{2}$ is amenable, note that the abelian subgroup generated by the word $b a$ is of index two.

\section{Proofs of the Main Results}

Our proofs of the above theorems will use a number of facts about the Stone-Cech compactification of $S, \beta S$, the products on $\beta S$, and some knowledge of ultrafilters.

First, recall that points in $\beta S$ can be identified with ultrafilters. Given $p \in \beta S$ if we let $\mathcal{N}(p)$ denote the set of open neighborhoods of $p$, then the subsets of $S$ defined by $\{U \cap G: U \in \mathcal{N}(p)\}$ is the ultrafilter that determines $p$. Conversely, if $p$ is an ultrafilter and $A \in p$, then $A^{-} \subseteq \beta S$ is a clopen set that is a neighborhood of $p$.

Recall that $\ell^{\infty}(S)=C(\beta S)$.

Next there are two ways to define products on $\beta S$. If $p, q \in \beta S$ and $p=$ $\lim _{\lambda} s_{\lambda}, q=\lim _{\mu} t_{\mu}$, then we set $p \cdot q=\lim _{\lambda}\left[\lim _{\mu} s_{\lambda} t_{\mu}\right]$ and $p \diamond q=\lim _{\mu}\left[\lim _{\lambda} s_{\lambda} t_{\mu}\right]$. These both define associative operations on $\beta S$ that make it into a semigroup. Moreover, $p \cdot q$ is continuous in the $p$ variable, while $p \diamond q$ is continuous in the $q$ variable. See [4]. Note that $s \cdot q=s \diamond q$ and $p \cdot s=p \diamond s$ when $s \in S$. 
These products on $\beta S$ induce associate actions of $\beta S$ on $\ell^{\infty}(S)=C(\beta S)$. Given $f \in C(\beta S)$ and $q \in \beta S$, if we define $f_{q}, f^{q}$ by $f_{q}(p)=f(q \diamond p)$ and $f^{q}(p)=f(p \cdot q)$, then $f_{q}, f^{q} \in C(\beta S)$. If we set $r_{q}(f)=q \cdot f=f^{q}$, then $q \rightarrow q \cdot f$ defines an associative, left action of the semigroup $(\beta S, \cdot)$ on $\ell^{\infty}(S)=C(\beta S)$. While setting, $l_{q}(f)=f \diamond q=f_{q}$ defines an associative, right action of the $\operatorname{semigroup}(\beta G, \diamond)$ on $\ell^{\infty}(S)=C(\beta S)$.

Lemma 7. Let $f \in \ell^{\infty}(G)$, let $s_{\mu} \in S, q \in \beta S$ and let $s_{\mu} \rightarrow q$. Then $s_{\mu} \cdot f_{\mu} \rightarrow q \cdot f$ and $f \diamond s_{\mu} \rightarrow f \diamond q$ in the weak ${ }^{*}$-topology.

Proof. Since $t \cdot q=\lim _{\mu} t s_{\mu}$ in the topology on $\beta G$, and $f$ is continuous, we have that $f(t \cdot q)=\lim _{\mu} f\left(t s_{\mu}\right)$. Thus, the net of elements $s_{\mu} \cdot f \in \ell^{\infty}(G)$ converges pointwise and boundedly to $q \cdot f$ and hence converges in the weak*-topology. The other proof is similar.

We are now ready to prove Theorem 1 .

Proof. It will suffice to prove the theorem in the case where $f \geq 0$. Since $\mathcal{C}(f)$ is compact in the weak*-topology and the action of left and right translation is continuous, by Zorn's lemma there will exist minimal non-empty weak*-compact, convex subsets of $\mathcal{C}(f)$ that are right and left translation invariant. Our goal is to prove that if $\mathcal{C}_{0}$ is such a subset, then every function in $\mathcal{C}_{0}$ is constant. First note that if $f_{1} \in \mathcal{C}_{0}$, then $\left\{f_{2} \in \mathcal{C}_{0}:\left\|f_{2}\right\| \leq\left\|f_{1}\right\|\right\}$ is weak*-closed and convex and right-left translation invariant. Hence, by minimality it is equal to $\mathcal{C}_{0}$. This forces $\left\|f_{1}\right\|=M$ to be constant on $\mathcal{C}_{0}$. Also, since every function in $\mathcal{C}(f)$ is positive, we have that $\sup _{s} f_{1}(s)=M$, for every $f_{1} \in \mathcal{C}_{0}$.

By [4, Theorem 1.51], $(\beta S, \cdot)$ contains a unique, non-empty minimal two-sided ideal denoted $K(\beta S)$. We claim that if $q \in K(\beta S)$ and $f_{1} \in \mathcal{C}_{0}$, then $f_{1}(q)=M$. Thus, $f_{1}$ attains its maximum at $q$.

Let us first show why this proves the theorem. Note that $\|f\|-\mathcal{C}_{0}=\left\{\|f\|-f_{1}\right.$ : $\left.f_{1} \in \mathcal{C}_{0}\right\}$ is a minimal, weak ${ }^{*}$-closed convex right-left translation invariant subset of $\mathcal{C}(\|f\|-f)$. Thus, by the above argument $\|f\|-f_{1}$ also achieves its maximum at $q$. Thus $f_{1}$ attains its maximum and minimum at $q$ which forces the function $f_{1}$ to be constant.

We now return to the proof of the claim. Assume to the contrary that $f_{1}(q)<M$. Pick $m, f_{1}(q)<m<M$ and let $U=\left\{p: f_{1}(p)<m\right\}$ be an open neighborhood of $q$. Hence, $A=U \cap S \in q$.

Given $A \subseteq S$, we let $b^{-1} A=\{t \in S: b t \in A\}$. Look at $B=\left\{b \in S: b^{-1} A \in q\right\}$. Note that $b \in B$ iff $b^{-1} \cdot(U \cap G) \in q$, iff $\left(b^{-1} U\right) \cap G \in q$ iff $q \in b^{-1} U$ iff $b \cdot q \in U$ iff $f_{1}(b \cdot q)<m$.

By [4, Theorem 4.39] the set $B$ is right syndetic. Hence, there exists a finite set $\left\{t_{1}, \ldots, t_{k}\right\}$ in $S$ such that $S=t_{1}^{-1} B \cup \cdots \cup t_{k}^{-1} B$.

Now let $f_{2}(s)=\frac{1}{k}\left[f_{1}\left(t_{1} s q\right)+\cdots+f_{1}\left(g t_{k} s q\right)\right]$. By the lemma, each function of $s, f_{1}\left(t_{i} s q\right)$ is a weak*-limit of left-right translates of $f_{1}$ and so is in $\mathcal{C}_{0}$. Hence, $f_{2} \in \mathcal{C}_{0}$. However, for $s \in S$, there exists $i$ so that $s \in t_{i}^{-1} B$. Thus, $b=t_{i} s \in B$. Hence, $f_{1}\left(t_{i} s q\right)=f_{1}(b q)<m$. From this it follows that $f_{2}(s) \leq \frac{1}{k}[(k-1) M+m]$ for every $g s \in S$.

Thus, $f_{2} \in \mathcal{C}_{0}$ and $\left\|f_{2}\right\|<M$, a contradiction. This contradiction completes the proof. 
We now are set to prove Theorem 2 .

Proof. As before it is enough to consider the case of a positive function. Proceeding as in the proof of the above theorem, we let $\mathcal{C}(f)$ denote the weak*-closed convex hull of the set of right translates of $f$, and arguing as before we take a minimal nonempty weak ${ }^{*}$-closed convex subset $\mathcal{C}_{0}$ that is invariant under right translations. For $f_{1} \in \mathcal{C}_{0}$ and $q \in K(\beta S)$, we show that $f_{1}$ attains its maximum value at $q$.

If we suppose that it does not attain its maximum at $q$ and define $A$ and $B$ as in the previous proof, we have that $B$ is right syndetic and hence also left syndetic.

Thus, there exists $\left\{t_{1}, \ldots, t_{k}\right\}$ such that $S=B t_{1}^{-1} \cup \cdots \cup B t_{k}^{-1}$. Now if $s \in S$, then $b=s t_{i}$ for some $b \in B$ and some $i$. Hence, $f_{1}\left(s t_{i} q\right)=f_{1}(b q)<m$. Setting $f_{2}(s)=$ $\frac{1}{k}\left[f_{1}\left(s t_{1} q\right)+\cdots+f_{1}\left(s t_{k} q\right)\right]$, we have that $f_{2} \in \mathcal{C}_{0}$ and $\left\|f_{2}\right\| \leq \frac{1}{k}[(k-1) M+m]<M$, a contradiction.

Thus, $f_{1}$ attains its maximum at $q$ and, as before, $\|f\|-f_{1}$ also attains its maximum at $q$, from which it follows that $f_{1}$ is constant.

We believe that our techniques should shed some light on a problem in the algebra of the Stone-Cech compactification. Given an infinite discrete semigroup $S$ the semigroups $(\beta S, \cdot)$ and $(\beta S, \diamond)$ each have minimal two-sided ideals, $K(\beta S, \cdot)$ and $K(\beta S, \diamond)$. It is known [4, Theorem 13.41] that $K(\beta S, \cdot)^{-} \cap K(\beta S, \diamond) \neq \emptyset$ and $K(\beta S, \cdot) \cap K(\beta S, \diamond)^{-} \neq \emptyset$, where $B^{-}$denotes the closure of a set in $\beta S$. Many examples are known for which $K(\beta S, \cdot) \cap K(\beta S, \diamond) \neq \emptyset$. The question of whether or not this intersection could ever be empty was raised in [4, page 241]. This question was answered in [1, where the first example of a semigroup for which the intersection is empty is given. Currently, there is little known about how the property that this intersection is either empty or non-empty relates to other properties of semigroups. We believe that the following conjectures are true.

Conjecture 8. Let $S$ be an infinite, discrete semigroup. If $K(\beta S, \cdot) \cap K(\beta S, \diamond) \neq \emptyset$, then $S$ is an amenable semigroup.

In particular, we believe that the following is true, which is sufficient to prove the above conjecture.

Conjecture 9. Let $S$ be an infinite, discrete semigroup. If $K(\beta S, \cdot) \cap K(\beta S \diamond) \neq \emptyset$ and $\mathcal{C}$ is a weak ${ }^{*}$-closed, convex subset of the cone of positive functions in $\ell^{\infty}(S)$ that is right translation invariant and is minimal among all such subsets, then every function in $\mathcal{C}$ attains its maximum at every point in this intersection.

\section{ACKNOWLEDGMENT}

The author is grateful to Tony Lau for several valuable observations.

\section{REFERENCES}

[1] S. Burns, The existence of disjoint smallest ideals in the two natural products on $\beta S$, Semigroup Forum 63 (2001), no. 2, 191-201. MR.1830683 (2002d:22003)

[2] M.M. Day, Amenable semigroups, Illinois J. Math. 1 (1957), 509-544. MR0092128(19:1067c)

[3] E. Granirer and Anthony T. Lau, Invariant means on locally compact groups, Illinois J. Math. 15 (1971), 249-257. MR0277667 (43:3400) 
[4] Neil Hindman and Dona Strauss, Algebra in the Stone-Cech Compactification, de Gruyter Expositions in Mathematics, Volume 27, Walter de Gruyter, New York, 1998. MR 1642231 (99j:54001)

[5] T. Mitchell, Constant functions and left invariant means on semigroups, Trans. Amer. Math. Soc. 119 (1965), 244-261. MR0193523 (33:1743)

Department of Mathematics, University of Houston, Houston, Texas 77204-3476

E-mail address: vern@math.uh.edu 